have been recently described. We report herein five cases of a unique and rare subtype of GI indolent T-cell lymphoma that has not been recognized in the current WHO classification.

Methods: Hematoxylin and eosin-stained sections of the five cases were reviewed. Immunohistochemical studies as well as in situ hybridization for Epstein-Barr virus-encoded small RNAs (EBER) were performed on formalin-fixed, paraffin-embedded tissue sections using a BechMark XT automated immunostainer. In four cases with available tissue, PCR-based analysis for the rearrangement of the T-cell receptor (TCR) genes was also performed.

Results: There were one female and four male patients, with a median age of 52 years (range, 29 to 66 years). The most common presenting symptoms included abdominal pain or distention, diahhrea, dyspepsia, and weight loss. Gastric body was the most commonly involved site, followed by colorectum and small intestine. Three patients had involvement of only one site, whereas an extensive whole-bowel involvement or disseminated disease was noted in the other two patients. Endoscopically, the lesions presented with multiple or solitary polyps or nodules, diffuse finely nodular lesions throughout the bowels, and alternatively, giant ulcerating masses. Staging workup revealed the lesions were restricted to the GI tract in most cases at diagnosis. Histologically, the lesions were characterized by either non-destructive infiltration of the lamina propria, or sometimes, transmural massive infiltration of the entire wall, by small to medium-sized atypical T-cells with a similar phenotype of CD3+, CD4-, CD5+, CD8-, CD30-, CD56-, TIA1+, EBER-. All lesions featured an extremely low proliferation activity reflected by the Ki-67 index. The molecular studies for the TCR gene rearrangement confirmed a clonal lymphoproliferative disorder in all four cases examined. Except for one patient was managed by a 'watch and wait' approach, four of the five patients were treated with CHOP-based chemotherapy due to an initial diagnosis of peripheral T-cell lymphoma, among whom two patients with giant ulcerating lesions also received a prior total or subtotal gastrectomy to reduce the risk that might caused potentially by chemotherapy. The two patients treated merely with chemotherapy showed little response and were both alive with persistent disease at last follow-up, whereas the other two patients received combined surgery and chemotherapy kept well without disease during follow-up.

Conclusions: We suppose the currently reported lesions might represent a novel subtype of peripheral T-cell lymphomas featuring a peculiar indolent clinical course. These lesions display clinicopathological features similar to but not identical to those described recently by Jaffe and Chan. Awareness of these indolent T-cell lymphoproliferative disorders of the GI tract is important for the correct diagnosis and optimal management.

\section{THE EXPRESSION AND ROLE OF CDK4 IN RHABDOMYOSARCOMA}

Chunxia Liu ${ }^{1,2}$, Yuanyuan Wang ${ }^{1}$, Yuwen Xie ${ }^{1}$, Bingcheng $\mathrm{Li}^{1}$, Yunzhao Chen $^{1,2}$, Xiaobin Cui ${ }^{1,2}$, Yan $\mathrm{Qi}^{1,2}$, Yan Ren ${ }^{1}$, Feng Li ${ }^{1,2}$

${ }^{1}$ Department of Pathology, Shihezi University School of Medicine, Shihezi, Xinjiang, PR China; and ${ }^{2}$ Department of Pathology, the First Affiliated Hospital, Shihezi University School of Medicine, Shihezi, Xinjiang, PR China

Background: Rhabdomyosarcoma (RMS) is the most common soft tissue sarcoma among children and adolescents, which includes two major subtypes, embryonal RMS (ERMS) and alveolar RMS (ARMS). Previous research has shown that CDK4 gene showes amplification and gain in RMS.

Aims: We detected CDK4 protein and mRNA expression in RMS, and analyzed its clinicopathological variable in RMS.

Methods: We investigated the expression of CDK4 in 45 cases of RMS and 36 cases of normal muscle controls by immunohistochemistry (IHC) using tissue microarrays and quantitative real-time polymerase chain reaction (QRT-PCR), respectively.

Results: The mean mRNA level of CDK4 in RMS samples was 4.53-fold of those in normal muscle tissues, indicating that the expression of CDK4 mRNA in RMS were significantly higher than those in normal control group. In addition, the rates of CDK4 protein expression in RMS, ARMS, and ERMS was $48.89 \%, 65 \%$, and $34.78 \%$, suggesting that difference in CDK4 protein expression exists among different RMS types ( $p=0.048$ ). Moreover, CDK4 protein expression was significantly associated with lymph node metastasis $(p=0.026)$.

Conclusions: These data suggest that CDK4 may be associated with the progression and metastasis of RMS.

\section{A RETROSPECTIVE STUDY TO CLASSIFY BREAST CARCINOMAS ACCORDING TO MOLECULAR CLASSIFICATION AND TO CORRELATE THE ER, PR, HER2 RESULTS WITH THE NOTTINGHAM GRADE AND THE AXILLARY LYMPH NODE STATUS AND TO COMPARE THE NOTTINGHAM GRADE WITH AXILLARY LYMPH NODE STATUS IN BREAST CARCINOMAS}

Indika Liyanage, Leonardo D. Santos, Jim Yong Sydney South West Pathology Services (SSWPS), Liverpool, NSW, Australia

Background: Breast carcinoma (BC) is a heterogeneous disease. Gene expression analyses identify several subtypes which are of prognostic and predictive importance.

Aims: (1) to determine the prevalence of BC subtypes from SSWPS area patients according to molecular classification using immunohistochemistry (IHC) as surrogate markers and (2) to determine the relationship between certain pathological characteristics.

Methods: We reviewed 312 cases of BC diagnosed in 2013 in our department. Molecular classification of $\mathrm{BC}$ was based on Estrogen Receptor (ER), Progesterone Receptor (PR), HER2/neu (HER2), HER2 CISH and Ki-67 results. Triple negative cases were subdivided into Basal-like and Unclassified groups using CK5/6, CK14 and EGFR. The ER, PR and HER2 results were correlated with Nottingham grade (NG) and axillary lymph node (ALN) status.

Results: The commonest molecular subtype was Luminal A subtype (45\%) followed by Luminal B (38\%) [Luminal B HER2 negative $(32 \%)$ and Luminal B HER2 positive (6\%)], HER2 positive (7\%), Basal-like (9\%) and Unclassified subtype (2\%). Positive ER and PR findings were inversely correlated with higher NG $(p \leq 0.0001)$ whereas high NG was positively correlated with positive HER2 status $(p \leq 0.0001)$. There was no correlation between ALN status and ER, PR and HER2 results. A statistically significant correlation was found between higher NG and number of ALN metastasis. Majority of Luminal A subtype BCs were either of NG 1 or 2 (135 cases, 96.4\%). Amongst the 
NG 3 cases, majority of the BCs belonged to Luminal B HER2 negative subtype (44.14\%).

Conclusion: This study shows that molecular classification of $\mathrm{BC}$ by IHC is practical and is necessary for therapeutic decision and prognosis as molecular testing is not always feasible in routine laboratories.

\section{DIAGNOSTIC AND PROGNOSTIC UTILITY OF MYB GENE REARRANGEMENT DETECTED BY FLUORESCENT IN-SITU HYBRIDISATION (FISH) IN ADENOID CYSTIC CARCINOMA}

Peter P. Luk ${ }^{1}$, Christina I. Selinger ${ }^{1}$, Timothy J. Eviston ${ }^{2}$, Rafael Ekmejian ${ }^{3}$, Denise $\mathrm{Foo}^{3}$, Jessica $\mathrm{Tay}^{3}$, Kan $\mathrm{Gao}^{2}$, Sydney Ch'ng ${ }^{2,4,5}$, Sandra A. O'Toole ${ }^{1,4,5}$, Jonathan R. Clark ${ }^{2,3,4,5}$, Ruta Gupta ${ }^{1,4,5}$

${ }^{1}$ Tissue Pathology and Diagnostic Oncology, Royal Prince Alfred Hospital, ${ }^{2}$ The Sydney Head and Neck Cancer Institute, ${ }^{3}$ University of New South Wales, Sydney, ${ }^{4}$ Central Clinical School, The University of Sydney, and ${ }^{5}$ Chris O'Brien Lifehouse, Sydney, Australia

Background: MYB rearrangement has been described in a proportion of adenoid cystic carcinoma (ACC).

Aims: This study evaluates the diagnostic and prognostic utility of MYB rearrangement in ACC.

Methods: Salivary gland malignancies at the Sydney Head and Neck Cancer Institute (1989-2014) were reviewed. Histopathologic evaluation, and fluorescent in-situ hybridization (FISH) studies were performed on ACCs and their morphologic mimics. Clinical follow-up was obtained.

Results: 39 cases of ACC were identified with approximately $50 \%$ arising within the parotid gland. The age range was 19 to 80 years (median: 45 years). The tumor size ranged from $9-44 \mathrm{~mm}$ (mean: 22mm). There were 3 locoregional recurrences, 7 distan metastases and 8 ACC related deaths seen over a 22 -year followup period. Nodal metastases, although uncommon, were the most significant predictor of disease specific survival $(p=0.019) .34$ cases were suitable for FISH and 16 (47\%) cases showed MYB rearrangement. MYB rearrangement was not seen in any cases of polymorphous low grade adenocarcinoma or epithelial myoepithelial carcinoma. MYB rearrangement was not seen in any of the high grade ACC or those with high grade transformation. MYB rearrangement was seen in $5(50 \%)$ patients with recurrence/metastases and in $11(45 \%)$ of those without

Conclusions: FISH for MYB rearrangement has high specificity but low sensitivity for ACC. MYB rearrangement did not provide prognostic information in this cohort.

\section{ANALYSIS OF KIT AND BRAF MUTATIONS IN CHINESE MELANOMA}

Jiaojie Lv, Yunyi Kong, Xu Cai, Xiaoyan Zhou Fudan University Shanghai Cancer Center, China

Background: Malignant melanoma is a rare disease in Asia, and knowledge of the status and the clinical significance of KIT and BRAF mutations in the Asian population is limited.

Aims: This study is designed to investigate the mutation status of KIT and BRAF in Chinese melanoma patients.
Methods: 144 cases of melanoma were analyzed for KIT mutations (exon9, 11, 13, 17) and BRAF (V600E, V600K, V600R) mutations by DNA sequencing using paraffinembedded tissues.

Results and conclusions: There were $36.1 \%$ of acral lentiginous melanoma (ALM), $24.3 \%$ of mucosal melanoma (MCM), $4.8 \%$ of melanoma on skin with chronic sun-induced damage (CSD), $25.0 \%$ of melanoma on skin without chronic sun-induced damage (non-CSD), and others were unclassifiable disease. The overall incidence of somatic mutations within the BRAF and KIT genes was $25 \%$ and $9.0 \%$, respectively. The incidence of BRAF mutation in acral, mucosa, CSD and non-CSD MM tissues was $15.4 \%$ (8/52), $2.9 \%$ (1/35), 57.1\% (4/7) and 50.0\% (18/36), respectively. Among the 36 patients with BRAF mutations, $88.9 \%$ (32/36) had V600E mutations. Melanomas with chronic sun-induced damage (CSD) were more likely $(57.1 \%)$ to show BRAF mutations. KIT mutation in acral, mucosa, CSD and non-CSD MM tissues was $7.7 \%$ (4/52), $20 \%$ (7/35), $14.3 \%(1 / 7)$ and $2.8 \%$ (1/36), respectively. Mucosal melanoma were most likely $(20 \%)$ to show KIT mutations. Among the 13 patients with KIT mutations, one had mutation in exon 9,9 in exon 11 and 3 in exon 13. The most prevalent type in BRAF mutation is V600E while that in KIT mutation is p.L576p of exon 11. They could be considered as potential drug targets in advanced melanoma therapy.

\section{TERMINAL DEOXYNUCLEOTIDYL TRANSFERASE (TDT)-NEGATIVE T-CELL LYMPHOBLASTIC LYMPHOMA DOES EXIST: A CASE REPORT}

$\mathrm{Li} \mathrm{Ma}^{1}$, Morag Whyte ${ }^{2}$, David Gillis ${ }^{3}$, Gael E. Phillips ${ }^{1}$, Olivier Ramuz

${ }^{1}$ Anatomical Pathology and Cytopathology, ${ }^{2}$ Paediatric Oncology, Lady Cilento Children Hospital, South Brisbane, and ${ }^{3}$ Immunology Pathology Queensland Central Laboratory Royal Brisbane and Women's Hospital, Herston, Queensland, Australia

Background: T-cell acute lymphoblastic leukaemia/lymphoma (T-ALL/LBL) is an aggressive neoplasm derived from T-cell progenitors that represents approximately $15 \%$ of childhood ALL cases. In conjunction with other features, TDT-positivity is considered as a hallmark for ALL/LBL. We report a case of TDT-negative T-LBL.

Case report: A 6 months-old male patient presented for an isolated anterior mediastinal mass with airway compression and elevated LDH. Histological assessment of needle core biopsy showed sheets of intermediate lymphocytes with monomorphic nuclei and high $\mathrm{N}: \mathrm{C}$ ratio. Most tumour cells showed central eosinophilic nucleoli or small peripheral nucleoli. Apoptotic cells and mitoses were frequent. Immunohistochemistry showed expression of CD45, CD99, CD2, CD3, CD5, CD8, and BCL-2. Ki67 proliferation index was $80 \%$. AE1/AE3, CD20, CD10, CD4, myeloperoxidase, EBER-ISH, and TDT were negative. Flow cytometry was concordant and detected surface alpha-beta TCR chains. Monoclonal rearrangement of both TCR-Gamma and TCR-Beta genes was detected. The features were consistent with T-LBL, medullary stage.

Discussion: T-ALL/LBL typically presents with blastic features. The mature morphology observed in this case may represent effect of steroids prior to biopsy. Cases of TDT-negative T-ALL/ LBL are rare. T-LBL, medullary stage is the most mature stage of thymic development, for which lack of TDT expression is 